\title{
ON THE LORENTZIAN TRANSFORMATION AND THE RADIATION FROM A MOVING ELECTRON.
}

\author{
BY DR. F. R. SHARPE.
}

(Read before the Amerioan Mathematical Society, February 29, 1908.)

1. Introduction. The electromagnetic field produced by a moving charge has been discussed by many writers.* Langevin $\dagger$ in particular has given an elegant investigation of the field due to a moving electron. Poincaré $\$$ has partially solved Langevin's problem by the Lorentzian $\S$ transformation. In the present paper the Lorentzian transformation is applied to the expressions for the scalar and vector potentials in the form of definite integrals instead of to the differential equations for the electromagnetic field. The field due to an electron whose velocity is zero at the instant considered is very simply determined. By means of the Lorentzian transformation, the field for the more general case when the velocity is not zero is deduced. The results are Langevin's expression for the electric force and a new expression for the magnetic force.

2. The electromagnetic equations and their solution. Let $E$ denote the electric force, $H$ the magnetic force, $\rho$ the volume density, and $v$ the velocity of the electrons, and let the velocity of light be the unit of velocity. Lorentz's $\|$ fundamental equations are

$$
\begin{array}{cc}
\operatorname{div} E=\rho, & \text { (2) } \quad \operatorname{div} \rho v=-\frac{\partial \rho}{\partial t}, \\
\operatorname{curl} H=\frac{\partial E}{\partial t}+\rho v, & \text { (4) } \quad \operatorname{curl} E=-\frac{\partial H}{\partial t} .
\end{array}
$$

The solution of these equations is known to be reducible to the determination of a scalar potential $\phi$ and a vector potential $\alpha$ which satisfy the equations

\footnotetext{
* See Lorentz, Encyklopädie, d. math. Wissensch., vol. V. 2, p. 174.

† Journal de Physique, 1905.

$\ddagger$ Circolo mat. di Palermo, 1906.

§Lorentz, Amsterdam Proc., 1903. Einstein, Annalen der Physik, 1905.

II Loc. cit.
} 


$$
\nabla^{2} \phi-\frac{\partial^{2} \phi}{\partial t^{2}}=-\rho
$$

where

$$
E=-\frac{\partial \alpha}{\partial t}-\operatorname{grad} \phi \quad \text { and }
$$

The solutions of (5) and (6) are known to be *

$$
\begin{aligned}
& \phi=\frac{1}{4 \pi} \iiint \frac{\rho_{1} d x_{1} d y_{1} d z_{1}}{r}, \\
& \alpha=\frac{1}{4 \pi} \iiint \frac{\rho_{1} v_{1} d x_{1} d y_{1} d z_{1}}{r},
\end{aligned}
$$

where $\rho_{1}$ and $v_{1}$ are the values of $\rho$ and $v$ at $\left(x_{1}, y_{1}, z_{1}\right)$ at time $t-r, r$ being the time from $(x, y, z)$ to $\left(x_{1}, y_{1}, z_{1}\right)$. These expressions for the potentials may be expressed in terms of the actual charges of the electrons. If we imagine a sphere whose center is $(x, y, z)$ and whose radius $r$ diminishes with the velocity of light and is zero at time $t$, an electron will affect $(x, y, z)$ only while the surface of the sphere is passing through the electron. Hence if the radial velocity of an electron is $v_{r}$, the effect of the motion of the electron is to increase the apparent charge in the ratio 1 to $1-v_{r}$. Therefore if de denotes the actual charge of an electron at $\left(x_{1}, y_{1}, z_{1}\right)$,

$$
\phi=\int \frac{d e}{r\left(1-v_{r}\right)}, \quad \text { (12) } \quad \alpha=\int \frac{v d e}{r\left(1-v_{r}\right)} .
$$

3. The Lorentzian Transformation. Consider the transformation

$$
x^{\prime}=\beta(x-V t), \quad t^{\prime}=\beta(t-V x), \quad\left(\beta=1 / \sqrt{\left.1-V^{2}\right)}\right.
$$

and its inverse

$$
x=\beta\left(x^{\prime}+V t^{\prime}\right), \quad t=\beta\left(t^{\prime}+V x^{\prime}\right) .
$$

If we wish to transform the electrical system into a new electrical system with the same charges, we must have

$$
\iiint \rho^{\prime} d x^{\prime} d y d z=\iiint \rho d x d y d z .
$$

\footnotetext{
* Rayleigh, Theory of sound, vol. 2, p. 104.
} 
On the left hand side of this equation $t^{\prime}$ is constant, while on the other side $t$ is constant. On account of the velocity $v_{x}$ of the electrons $\left(x+d x+v_{x} d t, t+d t\right)$ is tranformed into $\left(x^{\prime}+d x^{\prime}, t^{\prime}\right)$. Hence from (13)

$$
d x^{\prime}=\beta\left(d x+v_{x} d t-V d t\right), \quad 0=\beta\left\{d t-V\left(d x+v_{x} d t\right)\right\} .
$$

Solving, we find

$$
d x^{\prime}=\frac{d x}{\beta\left(1-V v_{x}\right)}
$$

and therefore to satisfy (15) we must have

$$
\rho^{\prime}=\beta \rho\left(1-V v_{x}\right) \text {. }
$$

It is important to notice that (13) and (16) completely determine the new electrical system.

We proceed to find the values of $v^{\prime}, \phi^{\prime}, \alpha^{\prime}, E^{\prime}, H^{\prime}$. Differentiating (13) we have

$$
\begin{gathered}
v_{x}^{\prime}=\frac{d x^{\prime}}{d t^{\prime}}=\frac{v_{x}-V}{1-V v_{x}}, \quad v_{y}^{\prime}=\frac{d y}{d t^{\prime}}=\frac{v_{y}}{\beta\left(1-V v_{x}\right)}, \\
v_{z}^{\prime}=\frac{d z}{d t^{\prime}}=\frac{v_{z}}{\beta\left(1-V v_{x}\right)}
\end{gathered}
$$

It is easily verified that the values of $\rho^{\prime}$ and $v^{\prime}$ just obtained satisfy the continuity equation

$$
\operatorname{div} \rho^{\prime} v^{\prime}=-\frac{\partial \rho^{\prime}}{\partial t^{\prime}}
$$

It should also be noticed that in (11) and (12)

$$
t-t_{1}=r
$$

or

$$
\left(t-t_{1}\right)^{2}=\left(x-x_{1}\right)^{2}+\left(y-y_{1}\right)^{2}+\left(z-z_{1}\right)^{2} .
$$

This equation is transformed by (14) into

$$
\left(t^{\prime}-t_{1}^{\prime}\right)^{2}=\left(x^{\prime}-x_{1}^{\prime}\right)^{2}+\left(y-y_{1}\right)^{2}+\left(z-z_{1}\right)^{2}
$$

or

$$
t^{\prime}-t_{1}^{\prime}=r^{\prime}
$$

Hence the new electron is in position at time $t_{1}^{\prime}$ to act on the 
new point $\left(x^{\prime}, y^{\prime}, z^{\prime}\right)$ at time $t^{\prime}$. Further it is readily shown from (17) and (18) that

$$
r^{\prime}\left(1-v_{r}^{\prime}\right)=\frac{r\left(1-v_{r}\right)}{\beta\left(1-V v_{x}\right)} .
$$

Hence

or

$$
\phi^{\prime}=\int \frac{d e}{r^{\prime}\left(1-v_{r}^{\prime}\right)}=\int \frac{\beta\left(1-V v_{x}\right) d e}{r\left(1-v_{r}\right)}
$$

and similarly

$$
\phi^{\prime}=\beta\left(\phi-V \alpha_{x}\right)
$$

$$
\alpha_{x}^{\prime}=\beta\left(\alpha_{x}-V \phi\right), \quad \alpha_{y}^{\prime}=\alpha_{y}, \quad \alpha_{z}^{\prime}=\alpha_{z} .
$$

Again from (14)

$$
\frac{\partial}{\partial x^{\prime}}=\beta\left(\frac{\partial}{\partial x}+V \frac{\partial}{\partial t}\right), \quad \frac{\partial}{\partial t^{\prime}}=\beta\left(\frac{\partial}{\partial t}+V \frac{\partial}{\partial x}\right) .
$$

Therefore

$$
E_{x}^{\prime}=-\frac{\partial \alpha_{x}^{\prime}}{\partial t}-\frac{\partial \phi^{\prime}}{\partial x^{\prime}}=-\frac{\partial \alpha_{x}}{\partial t}-\frac{\partial \phi}{\partial x}
$$

or

$$
E_{x}^{\prime}=E_{x}
$$

and similarly

$$
\begin{gathered}
E_{y^{\prime}}=\beta\left(E_{y}-V H_{z}\right), \quad E_{z^{\prime}}=\beta\left(E_{z}+V H_{y}\right), \quad H_{x^{\prime}}=H_{x}, \\
H_{y^{\prime}}=\beta\left(H_{y}+V E_{z}\right), \quad H_{z^{\prime}}=\beta\left(H_{z}-V E_{y}\right) .
\end{gathered}
$$

The new electrical system is therefore completely expressed in terms of the old system.

4. Radiation from an electron. If the charge of the electron is $e$ the potentials are, from (11) and (12),

$$
\phi=\frac{e}{4 \pi r\left(1-v_{r}\right)}, \quad \alpha=\frac{e v}{4 \pi r\left(1-v_{r}\right)} .
$$

Langevin has calculated the derivatives of $\phi$ and $\alpha$, and so determined the electric and magnetic forces. The problem is simplified by taking the $x$ axis in the direction of the velocity and applying the Lorentzian transformation so as to reduce the velocity to zero; solving the new system and reversing the 
transformation so as to obtain the solution of the original system. In this case

$$
V=v_{x}, \quad v_{y}=v_{z}=v_{x}^{\prime}=v_{y}^{\prime}=v_{z}^{\prime}=0
$$

and from (22)

$$
\phi^{\prime}=\frac{e}{4 \pi r^{\prime}}, \quad \alpha^{\prime}=0 .
$$

The derivatives of $\phi^{\prime}$ and $\alpha^{\prime}$ depend on the acceleration $j$. On differentiating (17) and using (23), we obtain

$$
j_{x}^{\prime}=\frac{d v_{x}^{\prime}}{d t^{\prime}}=\beta^{3} j_{x}, \quad j_{y}^{\prime}=\beta^{2} j_{y}, \quad j_{z}^{\prime}=\beta^{2} j_{z} .
$$

From (22)

$$
\phi^{\prime}+d \phi^{\prime}=\frac{e}{4 \pi\left(r^{\prime}+d r^{\prime}\right)\left(1-j_{r}^{\prime} d t_{1}^{\prime}\right)}, \quad d \alpha^{\prime}=\frac{e j^{\prime} d t_{1}^{\prime}}{4 \pi r^{\prime}} .
$$

But if $t^{\prime}$ is constant, (18) gives $d t_{1}^{\prime}=-d r^{\prime}$. Hence

$$
\operatorname{grad} \phi^{\prime}=-\frac{e}{4 \pi r^{\prime 3}} \bar{r}^{\prime}-\frac{e j_{r}^{\prime}}{4 \pi r^{2}} \bar{r}^{\prime}
$$

Also if $r^{\prime}$ is constant $d t_{1}^{\prime}=d t^{\prime}$. Hence

Hence

$$
\frac{\partial \alpha^{\prime}}{\partial t^{\prime}}=\frac{e j}{4 \pi r^{\prime}}
$$

$$
E^{\prime}=\frac{e}{4 \pi r^{\prime 3}} \bar{r}^{\prime}+\frac{e j_{r}^{\prime}}{4 \pi r^{\prime 2}} \bar{r}^{\prime}-\frac{\overline{j^{\prime}}}{4 \pi r^{\prime}}
$$

Again

$$
H^{\prime}=\operatorname{curl} \alpha^{\prime}=\frac{\partial \alpha_{t}^{\prime}}{\partial r^{\prime}}=\frac{e}{4 \pi r^{\prime 2}}\left[j^{\prime} r^{\prime}\right],
$$

where $d \alpha_{t}^{\prime}$ is the transverse component of $d \alpha^{\prime}$. The first term of $E^{\prime}$ is the force $E_{1}^{\prime}$ due to the electron at rest. The other two terms are due to the acceleration and have a resultant $e j_{t}^{\top} / 4 \pi r^{\prime}=E_{2}^{\prime}$. This is obviously equal and perpendicular to $H^{\prime}$. Hence $E_{2}^{\prime}$ and $H^{\prime}$ are equal and $r^{\prime}, E_{2}^{\prime}, H^{\prime}$ are mutually perpendicular, $E_{2}^{\prime}$ being in the plane of $r^{\prime}$ and $j^{\prime}$. If we now apply the inverse Lorentzian transformation the first term of $E^{\prime}$ gives the well-known field for a uniformly moving charge, 
the velocity wave of Langevin. The terms depending on the acceleration (when expressed in terms of the original coordinates the origin being taken at the electron so that $x_{1}=y_{1}=z_{1}=0$ ) are

$$
\begin{aligned}
& E_{x}^{\prime}=\frac{e}{4 \pi(r-V x)^{3}}\left\{\left(y j_{y}+z j_{z}\right)(x-V r)-\left(y^{2}+z^{2}\right) j_{x}\right\} \\
& E_{y}^{\prime}=\frac{e}{4 \pi \beta(r-V x)^{3}}\left[\left\{z j_{z}+\beta^{2}(x-V r) j_{x}\right\} y-\left\{z^{2}+\beta^{2}(x-V r)^{2}\right\} j_{y}\right], \\
& E_{z}^{\prime}=\frac{e}{4 \pi \beta(r-V x)^{3}}\left[\left\{\beta^{2}(x-V r) j_{x}+y j_{y}\right\} z-\left\{\beta^{2}(x-V r)^{2}+y^{2}\right\} j_{z}\right] \\
& H_{x}^{\prime}=\frac{e}{4 \pi(r-V x)^{2}}\left(z j_{y}-y j_{z}\right), \\
& H_{y}^{\prime}=\frac{e \beta}{4 \pi(r-V x)^{2}}\left\{(x-V r) j_{z}-z j_{x}\right\} \\
& H_{z}^{\prime}=\frac{e \beta}{4 \pi(r-V x)^{2}}\left\{y j_{x}-(x-V r) j_{y}\right\} .
\end{aligned}
$$

Making the transformation, we easily find

$$
\begin{aligned}
E_{x} & =\frac{e}{4 \pi(r-V x)^{3}}\left\{\left(x j_{x}+y j_{y}+z j_{z}\right)(x-V r)-r(r-V x) j_{x}\right\}, \\
E_{y} & =\frac{e}{4 \pi(r-V x)^{3}}\left[\left(x j_{x}+y j_{y}+z j_{z}\right) y-r(r-V x) j_{y}\right], \\
(27) E_{z} & =\frac{e}{4 \pi(r-V x)^{3}}\left\{\left(x j_{x}+y j_{y}+z j_{z}\right) z-r(r-V x) j_{z}\right\}, \\
H_{x} & =\frac{e}{4 \pi(r-V x)^{3}}\left(z j_{y}-y j_{z}\right)(r-V x), \\
H_{y} & =\frac{e}{4 \pi(r-V x)^{3}}\left\{\left(x j_{z}-z j_{x}\right)(r-V x)-\left(x j_{x}+y j_{y}+z j_{z}\right) V z\right\} . \\
H_{z} & =\frac{e}{4 \pi(r-V x)^{3}}\left\{\left(y j_{x}-x j_{y}\right)(r-V x)\right. \\
\quad & \left.\quad+\left(x j_{x}+y j_{y}+z j_{z}\right) V y\right\} .
\end{aligned}
$$

Expressed in vector notation, we have therefore

$$
E=\frac{e}{4 \pi\{r-(V r)\}^{3}}(r j) \bar{R}-\frac{e r}{4 \pi\{r-(V r)\}^{2}} \bar{j},
$$


which is Langevin's result, $R$ being the distance from $(x, y, z)$ to the point which the electron would reach at time $t$ if its acceleration were zero; that is, a force directed towards this point and a force in the direction of the acceleration. Moreover

$H=\frac{e}{4 \pi\{r-(V r)\}^{2}}[r j]-\frac{e}{4 \pi\{r-(V r)\}^{3}}(r j)[V r]$,

that is, a force perpendicular to $\bar{r}$ and $\bar{j}$ and a force perpendicu$\overline{\text { lar to }} \bar{r}$ and $\bar{V}$. It is easily verified from (27) that $E$ is equal to $H$ and that $r, E, H$ are mutually perpendicular.

Cornall University, January, 1908.

\section{SHORTER NOTICES.}

On the Resolution of Higher Singularities of Algebraic Curves into Ordinary Nodes. By B. M. WALKer. Doctor Dissertation, University of Chicago, 1906. 8 vo. $52 \mathrm{pp}$. + Vita.

As stated in the introduction, this dissertation completes in detail a procedure proposed by Clebsch for resolving the higher singularities of algebraic plane curves. The method is this : To relate the points of the plane, one to one, to points of a general cubic surface in such a way that one point of multiplicity higher than 2 , with tangents all distinct, is distributed into ordinary points of a curve on the cubic. Then by projecting back upon the plane from a center on the cubic surface, no new singularities are introduced except ordinary double points.

The first half of the work ( 26 pages) is devoted to the relation of plane and cubic surface by means of a three-parameter linear system of plane cubics with six common base points. The restriction that these six points shall not lie on a conic suffices to insure that there shall be in the plane no fundamental curves, $i$. $e$. no curves all of whose points correspond to a single point of the cubic. On the cubic surface, however, the six base points of the plane are represented by six straight lines. The author shows in detail what plane curves give rise to the rest of the twenty-seven lines on the cubic. Six of these are conics which contain five of the base points apiece; the 